\title{
An Aplysia Type 4 Phosphodiesterase Homolog Localizes at the Presynaptic Terminals of Aplysia Neuron and Regulates Synaptic Facilitation
}

\author{
Hyungju Park, Jin-A Lee, Changhoon Lee, Min-Jeong Kim, Deok-Jin Chang, Hyoung Kim, Seung-Hee Lee, \\ Yong-Seok Lee, and Bong-Kiun Kaang \\ National Research Laboratory of Neurobiology, Institute of Molecular Biology and Genetics, School of Biological Sciences, College of Natural Sciences, Seoul \\ National University, Seoul 151-742, Korea
}

\begin{abstract}
The cAMP-dependent signaling pathway is critically involved in memory-related synaptic plasticity. cAMP-specific type 4 phosphodiesterases (PDE4) play a role in this process by regulating the cAMP concentration. However, it is unclear how PDE4 is involved in regulating synaptic plasticity. To address this issue in Aplysia sensory-to-motor synapses, we identified a long isoform of the PDE4 homolog in Aplysia kurodai (apPDE), with genetic and biochemical properties similar to those of mammalian PDE4s. Furthermore, apPDE is localized to the membrane and presynaptic region. Both apPDE overexpression and knock-down impaired short- and long-term facilitation, indicating that an appropriate expression level of apPDE in synaptic regions is required for normal synaptic facilitation. By using fluorescence resonance energy transfer-based measurement of in vivo protein kinase A (PKA) activation, we found that the PKA activation by 5-hydroxytryptamine (5-HT) was impaired in both apPDE-overexpressed and knock-down synapses. Analogous to the inhibition of apPDE by RNA interference, chronic rolipram treatment before 5-HT stimulation also impaired the PKA activation by 5-HT, suggesting that regulation of the synaptic cAMP level by PDE4 is critical for normal synaptic facilitation. Together, we suggest that PDE4s localized in the synapses play a critical role in regulating the optimum cAMP level required for normal synaptic plasticity.
\end{abstract}

Key words: PDE4; cAMP; rolipram; synaptic facilitation; FRET; memory

\section{Introduction}

cAMP is a ubiquitous second-messenger molecule and activates several downstream effectors such as the protein kinase A (PKA) holoenzyme. In the PKA pathway, a cAMP-specific phosphodiesterase (PDE) acts as a negative regulator by reducing the intracellular cAMP level (Soderling and Beavo, 2000). PDEs have been classified into the 11 families based on their amino acid sequence, substrate specificity, and sensitivity for biochemical activators or inhibitors (Soderling et al., 1998a,b, 1999; Fawcett et al., 2000; Francis et al., 2001; Mehats et al., 2002). Among these, the type 4 PDE family contains four subtype genes (PDE4A, PDE4B, PDE4C, and PDE4D) (Verghese et al., 1995). According to their structures, each subtype is classified as super-short, short, and long isoforms (Houslay and Adams, 2003). Long-form PDE4 is known to be targeted to membrane fractions or interact with its partner molecules, such as anchoring proteins (Shakur et al., 1993; Beard et al., 2002; Bolger et al., 2003; Conti et al., 2003).

\footnotetext{
Received May 17, 2005; revised Aug. 6, 2005; accepted Aug. 23, 2005.

This work was supported by the Korea Ministry of Science and Technology under National Research Laboratory Program Grant M1-0104-00-0140 and Brain Research Center-Frontier Program Grant M103KV010012 03 K2201 01210 and by the Marine and Extreme Genome Research Center Program, Ministry of Marine Affairs and Fisheries, Republic of Korea.

Correspondence should be addressed to Dr. Bong-Kiun Kaang, National Research Laboratory of Neurobiology, Institute of Molecular Biology and Genetics, School of Biological Sciences, College of Natural Sciences, Seoul National University, San 56-1 Silim-dong Kwanak-gu, Seoul 151-742, Korea. E-mail: kaang@snu.ac.kr.

DOI:10.1523/JNEUROSCI.1989-05.2005

Copyright $\odot 2005$ Society for Neuroscience $\quad$ 0270-6474/05/259037-09\$15.00/0
}

Therefore, this isoform is believed to regulate the spatial and temporal characteristics of cAMP gradients in synapses.

cAMP-dependent signaling has come into the spotlight as a memory regulator. Previous reports with type 1 adenylyl cyclase transgenic mice (Wang et al., 2004) and rolipram, a PDE4specific inhibitor (Barad et al., 1998), suggest that the elevation of cAMP level enhances synaptic plasticity and memory. In contrast, several conflicting studies, such as those on the dunce mutant in Drosophila (Dudai et al., 1976; Tully and Quinn, 1985; Chen et al., 1986; Dubnau and Tully, 1998) and $\mathrm{G}_{\mathrm{i}} \alpha$ knock-out mice (Pineda et al., 2004), reported the impaired learning and memory phenotype caused by the elimination of the cAMPnegative regulator. These results suggest that the optimum range of cAMP level and PKA activity are required for normal memory formation. However, the exact role of PDE4 in this process is not clear.

In Aplysia, the sensitization of gill and siphon withdrawal reflex and related short- and long-term synaptic facilitation represent a simple form of learning and memory. These processes are dependent on the increase of cAMP and activation of PKA in sensory neurons by neurotransmitter 5-hydroxytryptamine (5HT) (Castellucci et al., 1980, 1982; Bernier et al., 1982; Greenberg et al., 1987; Schacher et al., 1988; Byrne and Kandel, 1996). cAMP-specific PDEs are thought to be key elements in this process; however, little is known about the role of PDE4 in synaptic facilitation. In the present study, to investigate the role of PDE4 in 
synaptic plasticity between Aplysia sensory and motor neuron, we cloned a novel long isoform of the Aplysia PDE4 homolog (apPDE), which showed genetic structure, biochemical properties, and subcellular localization similar to those of mammalian long-isoform PDE4. Our results suggest that cAMP level in synaptic regions is regulated by apPDE and that this regulation is critical for short- and long-term synaptic facilitation in Aplysia.

\section{Materials and Methods}

Cloning. We obtained the apPDE fragment of Aplysia kurodai from the expressed sequence tagged (EST) database (pYESTrp2-based, 5'CAP and R5'CAP library) by using the basic local alignment search tool search. The super-short and short isoforms of apPDEs were identified before the discovery of the long isoform of PDE. In brief, we used a $1006 \mathrm{bp}$ apPDE fragment from EST as a probe to screen $\sim 6 \times 10^{5}$ clones of Aplysia kurodai cDNA libraries and isolated several clones encoding parts of apPDE. Based on the sequences of these clones, we obtained the full length of super-short and short isoforms of apPDE by performing PCR with specific primers. For the super-short form of apPDE, we used the following primers: PDE-XhoI-s, 5'-CA CTCGAG GCC ACC ACC ATG CCT TCT GT G TGC GCG-3'; PDE-BamHI-a, 5' -CG GGATCC TCA TTT CTT GTC CTG T CC- $3^{\prime}$. For the short form of apPDE, we performed PCR in pYESTrp2 library with designed primer sets: BcoIII-s, 5'-CGCACTTACATTCGCACTG-3'; PDE-2A, 5' -GTGGTCGACGTCATAAAT- $3^{\prime}$. PCR products were cloned into a pGEM-T vector (Promega, Madison, WI) and confirmed by sequencing.

For the cloning of the long isoform of apPDE, we used a $418 \mathrm{bp}$ region between the upstream conserved region 1 (UCR1) and UCR2 domain as a probe. We screened the R5'CAP library using this probe and found two different clones partially containing the long-form-specific N-terminal domain and the catalytic domains. We combined these partial clones to obtain full length clones. To confirm the full sequence of these clones, we designed two primers based on our full sequences: apPDE_RT-s, $5^{\prime}$ CTCTGCTCCGCGTCTTCTAT-3'; new PDE-a, 5'-TCATTTCTTGTCCTGTCCTCT- $3^{\prime}$. Using cDNA from the sensory cluster in the pleural ganglion as a template, we performed reverse transcription (RT)PCR with the above primer set. We obtained specific full-length PCR products and confirmed their sequence (see Fig. $2 A$ ).

Plasmid construction. For pcDNA3.1-apPDE-FLAG constructs, we performed PCR with PDE-BamHI-s and PDE-BamHI-a: 5'-CG GGA TCC TTA TTT TGT TTC ACC AG-3'. We inserted BamHI-digested PCR product into a BamHI-digested pcDNA3.1 vector (Invitrogen, San Diego, CA).

For the FLAG-tagged expression vector (pNEX $\delta$-apPDE-FLAG), we generated PCR products of apPDE by 30 cycles of PCR performed with the following primers: PDE-XhoI-s, 5'-GA CTC GAG GCC ACC ACC ATG CCT TCT GTG TGC GC-3'; PDE-BamHI-a, 5'-CG GGA TCC TCA TTT CTT GTC CTG TCC-3'. We inserted SalI-BamHI-digested PCR product into the pNEX $\delta$ vector, which was digested with SalI and BamHI.

For $\mathrm{pNEX} \delta$-apPDE $\Delta \mathrm{N}$-FLAG, we generated the PCR product from pNEX $\delta$-apPDE-FLAG by using the following primers: BamHI-apPDEdeltN-s, 5'-CG GGA TCC ATG CCC CAA AGG AGG GAG TCC- 3'; BamHI-LacZ-a, 5'-CG GGA TCC GAC ACC AGA CCA ACT GG.-3'. We ligated the BamHI-digested PCR product and pNEX $\delta$.

$P D E$ activity assay. We assayed PDE activity by a modified procedure of Thompson and Appleman (1971) and Bauer and Schwabe (1980). The principle of this method is that ${ }^{3} \mathrm{H}$-labeled cAMP is converted to ${ }^{3} \mathrm{H}$ labeled 5'-AMP by phosphodiesterase activity. Degraded ${ }^{3} \mathrm{H}$-labeled cAMP is purified with QAE-Sephadex (Sigma, St. Louis, MO) columns. We prepared the apPDE protein by overexpressing pcDNA3.1-apPDEFLAG in COS7 cells. We confirmed that the overexpressed apPDE protein mainly existed in the soluble part by Western blotting using monoclonal mouse anti-FLAG (Sigma) (data not shown). The assay consists of a two-step procedure. First, we performed reactions at pH 8.0 in $40 \mathrm{~mm}$ Tris- $\mathrm{HCl}$ buffer containing $0.5 \mathrm{mM} \mathrm{MgCl}_{2}, 1 \mu \mathrm{M}\left[{ }^{3} \mathrm{H}\right] \mathrm{cAMP}(80,000$ cpm), and diluted apPDE-overexpressed COS7 cytosolic extract into a final volume of $0.2 \mathrm{ml}$. We initiated the reaction by adding the extract to the assay mixture, performed in a $1.5 \mathrm{ml}$ tube at $30^{\circ} \mathrm{C}$ for $10 \mathrm{~min}$, and terminated by heating the tubes to $95^{\circ} \mathrm{C}$ for $3 \mathrm{~min}$, followed by immediate cooling on ice. In the second step, $50 \mu \mathrm{l}$ of Crotalus atrox snake venom $(1 \mathrm{mg} / \mathrm{ml})$ (Sigma) was added for $\left[{ }^{3} \mathrm{H}\right]$ adenosine formation. After 10 min of incubation at $30^{\circ} \mathrm{C}$, we terminated the reaction by applying an aliquot of $0.2 \mathrm{ml}$ to the QAE A-25 Sephadex column previously equilibrated with $3 \mathrm{ml}$ of $30 \mathrm{~mm}$ ammonium formate, $\mathrm{pH} \mathrm{6.0.} \mathrm{We} \mathrm{collected}$ elutes directly into scintillation vials and counted radioactivity in $8 \mathrm{ml}$ of a scintillation fluid. We determined dose-dependent inhibition in the presence of $1 \mu \mathrm{M}$ cAMP and over a range of $10 \mathrm{~nm}$ to $100 \mu \mathrm{M}$ rolipram (Sigma) and $1 \mu \mathrm{M}$ to $1 \mathrm{~mm}$ 3-isobutyl-1-methylxanthine (IBMX) (Sigma). PDE inhibitors (rolipram and IBMX) were dissolved in 100\% dimethylsulfoxide (DMSO) and diluted with the enzyme diluents. The final concentration of DMSO in the assay mixture was $1 \%$. We determined the $\mathrm{IC}_{50}$ from these values, using a nonlinear least-squares fitting algorithm in Origin 6 software (Microcal, Northampton, MA). We determined $K_{\mathrm{m}}$ values over a substrate range of $0.1-20 \mu \mathrm{M}$ cAMP (eight different concentrations) by fitting to the hyperbolic form of the Michaelis-Menten equation by using a least-square procedure in the Origin 6 software.

Immunocytochemistry and image analysis. We performed immunocytochemistry as described by Martin et al. (1997). The monoclonal mouse anti-FLAG antibody (Sigma) and the secondary cyanine 3 (Cy3)conjugated anti-mouse antibody (Amersham Biosciences, Piscataway, NJ) were used in dilution ratios of 1:250 and 1:1000, respectively. We obtained and analyzed fluorescence images by a confocal laser-scanning microscope (Radiance 2000; Zeiss, Jena, Germany) and NIH Image J software (National Institutes of Health, Bethesda, MD), respectively.

In vivo measurement of PKA activity. Plasmid constructs for in vivo cAMP level imaging were gifts from Manuela Zaccolo (University of Padua, Padua, Italy), and the method used for analysis was described previously (Zaccolo and Pozzan, 2002). In brief, because cAMP causes dissociation of the PKA regulatory and catalytic subunit by binding to the regulatory subunit, the elevated cAMP level can be detected by decreased fluorescence resonance energy transfer (FRET) between these subunits (Zaccolo and Pozzan, 2002). This reduced FRET also indicates the degree of PKA activation, because the dissociation of PKA regulatory subunit by cAMP binding produces free catalytic subunits that could phosphorylate their target proteins. Decreased FRET can be converted into an elevated cyan fluorescent protein (CFP)/yellow fluorescent protein (YFP) emission ratio. We performed FRET imaging on a Zeiss LSM510 confocal laser scanning microscope. RII (type II regulatory subunit)-CFP- and CAT (catalytic subunit)-YFP-expressed Aplysia sensory neurons were excited at $458 \mathrm{~nm}$, and fluorescence emission was collected with BP475525 (for CFP) and LP530 (for YFP) emission filters. To test whether FRET between RII-CFP and CAT-YFP probes occurred in Aplysia neuron, we used the acceptor bleaching method and calculated FRET efficiency as described previously (Karpova et al., 2003). After background correction, we measured both CFP and YFP emission values using the NIH Image J software, and the CFP/YFP ratios were subsequently calculated. 5-HT (Sigma) was freshly prepared by dissolving it in L15 (Sigma)/ artificial seawater (ASW). A $200 \mathrm{~mm}$ stock solution of 8-(4chlorophenylthio)-cAMP (8-CPT-cAMP; Sigma) in DMSO was diluted freshly by dissolving in L15/ASW and used. In the chronic rolipram experiment, we applied diluted rolipram into bath 24 and $48 \mathrm{~h}$ before FRET measurement. The final concentration of DMSO was below $0.1 \%$.

Statistics. Student's $t$ test and two-way ANOVA with Bonferroni's post hoc test was performed using Prism version 4.00 (GraphPad Software, San Diego, CA).

Cell cultures, electrophysiology, and the induction of long-term facilitation by 5-HT. We prepared both culture dishes and medium as described previously (Schacher and Proshansky, 1983; Montarolo et al., 1986). We isolated sensory cells from the pleural ganglia of adult animals (100-150 g) and cultured these for sensory neuron culture or cocultured these for sensory-to-motor culture with a left F cluster innervating to the siphon (LFS) motor neuron from the abdominal ganglia of adult animals, as described previously (Montarolo et al., 1986). Cultures were maintained for $3-4 \mathrm{~d}$ at $18^{\circ} \mathrm{C}$ and then used.

For the electrophysiological recording, the motor cell was then im- 
paled intracellularly with a glass microelectrode filled with $2 \mathrm{M} \mathrm{K}$-acetate, $0.5 \mathrm{M} \mathrm{KCl}$, and $10 \mathrm{mM} \mathrm{K}$-HEPES (10-15 M 2 ), and the membrane potential was maintained at $-30 \mathrm{mV}$ below its resting value. The EPSP was evoked in the LFS by stimulating the sensory neurons with a brief depolarizing stimulus with an extracellular electrode. To examine basal synaptic transmission, we measured the EPSP before and $48 \mathrm{~h}$ after microinjection of pNEX $\delta$-apPDE-FLAG or specific double-stranded RNA (dsRNA). To investigate the effect of apPDE on synaptic plasticity, we measured the initial EPSP value $24 \mathrm{~h}$ after microinjection. We then gave one pulse of 5-HT to induce short-term facilitation or five pulses of 5-HT for $5 \mathrm{~min}$ at $15 \mathrm{~min}$ intervals to induce long-term facilitation. The amount of synaptic facilitation was calculated as the percentage change in EPSP amplitude recorded after the 5-HT treatment versus its initial value before treatment. All data are presented as the mean \pm SEM percentage change in EPSP amplitude.

RNA synthesis by in vitro transcription. To prepare template DNAs for in vitro transcription, we inserted the cDNA for apPDE-specific fragment into a pLITMUS28i vector (New England Biolabs, Beverly, MA). We synthesized dsRNA by in vitro transcription of linearized template DNAs by using T7 RNA polymerase according to the instructions of the manufacturer (Ambion, Austin, TX).

\section{Results}

\section{apPDE is similar to mammalian long-form PDE4}

We obtained an EST clone homologous to the catalytic domain of the Drosophila dunce gene from the Aplysia EST database. By using this EST clone as a probe, we cloned full-length cDNA by library screening and nested PCR (see Materials and Methods).

The long isoform of mammalian PDE4 contains a unique $\mathrm{N}$-terminal domain and complete UCR1 and UCR2 domains as well as catalytic domains; however, the short and super-short isoforms contain partial UCR1 and UCR2 domains and only the UCR2 domain, respectively (Conti et al., 2003). Based on this, we found that the structure of the 792 aa candidate (apPDE) is most similar to that of the mammalian long isoform of PDE4 (supplemental Fig. $1 A, B$, available at www.jneurosci.org as supplemental material). We also found a high degree of similarity between the overall amino acid sequences of apPDE and mammalian PDE4D or dunce (dunce, 60\% identity and 73\% similarity; human PDE4D7, PDE4D8, and rat PDE4D7, 64\% identity and 77\% similarity) (supplemental Fig. $1 A$, available at www.jneurosci.org as supplemental material). Among PDE4 isoforms, the overall amino acid sequence homology was highest with PDE4D7 and PDE4D8 (long isoform of PDE4D), suggesting that apPDE is a PDE4D homolog.

apPDE also contained the domain for cAMP hydrolysis and canonical PKA phosphorylation sites that are conserved in the mammalian PDE4 and dunce (Sette and Conti, 1996) (supplemental Fig. $1 A$, available at www.jneurosci.org as supplemental material). These similar structural properties suggest that this clone is most probably similar to a long isoform of PDE4.

\section{apPDE showed cAMP-specific activity and rolipram-sensitive inhibition}

PDE4 is known to have substrate specificity for cAMP and to have lower $\mathrm{IC}_{50}$ values for rolipram, a PDE4-specific inhibitor, than for IBMX, a general PDE inhibitor (Houslay and Adams, 2003). To confirm whether apPDE has a PDE4-like phosphodiesterase activity, we performed the biochemical PDE activity assay to measure these features.

First, the phosphodiesterase activity of apPDE was only cAMP specific and showed no activity for cGMP (Fig. 1A). We found that the $K_{\mathrm{m}}$ value for cAMP was $5.7 \pm 0.7 \mu \mathrm{M}$ (mean $\pm \mathrm{SEM} ; n=$ 3 ). Second, the inhibitory effect of rolipram on cAMP-specific PDE activity was higher than that of IBMX (Fig. $1 B$ ). We found
A
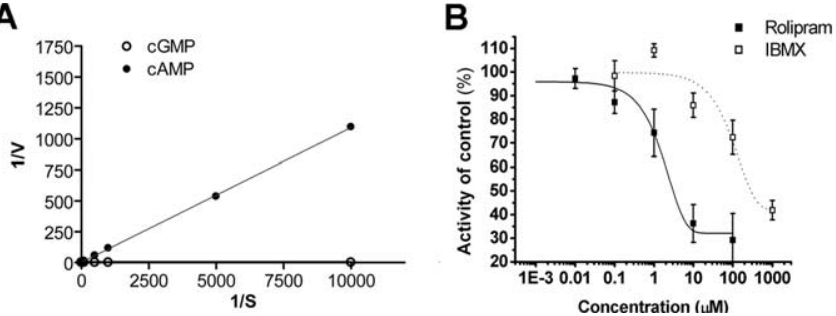

Figure 1. CAMP-specific PDE activity and rolipram-sensitive inhibition of PDE activity of apPDE. PDE activity assay was performed by a modified protocol from Thompson and Appleman (1971) and Bauer and Schwabe (1980) (see Materials and Methods). A, Representative plot of substrate specificity of apPDE. Enzyme activity was determined over a substrate concentration range of $0.1-20 \mu \mathrm{m}$ using the substrates CAMP and cGMP in eight different concentrations and fitted to the linear regression function. All three independent experiments demonstrated that apPDE hydrolyzed only CAMP but did not have activity for CGMP, indicating CAMP-specific PDE activity. The $K_{\mathrm{m}}$ value for cAMP was $5.7 \pm 0.7 \mu \mathrm{m}$ (mean $\pm \mathrm{SEM} ; n=3$ ). $\boldsymbol{B}$, Dose-dependent inhibition of apPDE. Inhibition of PDE activity was determined in the presence of $1 \mu \mathrm{m} \mathrm{CAMP}$ as a substrate and over a range of $10 \mathrm{~nm}$ to $100 \mu \mathrm{m}$ rolipram or $1 \mu \mathrm{m}$ to $1 \mathrm{~mm}$ IBMX. These values were plotted and fitted to nonlinear least-squares algorithm. Values are the mean \pm SEM of the percentage of the relative PDE activity from three independent experiments. Compared with IBMX, rolipram inhibits CAMP phosphodiesterase activity more sensitively $\left(\mathrm{IC}_{50}\right.$ value for rolipram, $4.0 \pm 1.8 \mu \mathrm{m}, n=3 \mathrm{vs} \mathrm{IC}_{50}$ value for IBMX, 188.6 $\pm 13.6 \mu \mathrm{m}, n=3$ ).

that the $\mathrm{IC}_{50}$ values for rolipram and IBMX were $4.0 \pm 1.8 \mu \mathrm{M}$ $($ mean \pm SEM; $n=3)$ and $188.6 \pm 13.6 \mu \mathrm{M}$ (mean \pm SEM, $n=$ $3)$, respectively. Considered together, we conclude that apPDE has biochemical features similar to those of mammalian PDE4s.

\section{apPDE was targeted to presynaptic regions}

Previous studies suggest that long-form PDE4 is mainly localized to the membrane fraction in non-neuronal cells, and its unique $\mathrm{N}$-terminal domain directs this localization as well as interaction with its partners (Shakur et al., 1993; Beard et al., 2002; Bolger et al., 2003). However, little is known about the cellular localization of PDE4 in neurons. Therefore, we examined the cellular distribution of apPDE in sensory neurons.

First, the existence of apPDE mRNA in Aplysia sensory neurons was revealed by RT-PCR performed with a specific primer set spanning the full length of apPDE (Fig. $2 A$ ). Next, we overexpressed FLAG-tagged apPDE to examine the cellular localization of PDE4. Immunocytochemistry data indicated that apPDEFLAG is mainly localized to the membrane region in soma and neurites (Fig. $2 B$ ). In particular, overexpressed apPDE was highly enriched in neurite ends and varicosity-like regions (Fig. 2B), suggesting that apPDE is targeted to synaptic regions in distal neurite regions. To further examine the synaptic localization of apPDE more clearly, we used a presynaptic marker, synaptophysin-enhanced green fluorescent protein (EGFP) (Kim et al., 2003). When apPDE-FLAG was coinjected with synaptophysin-EGFP into sensory neurons, most apPDE-FLAG puncta were colocalized with the synaptophysin-EGFP puncta (Fig. 2C). We also confirmed the synaptic localization of apPDE by using Aplysia sensory-to-motor synapse cultures and by analyzing the colocalization patterns (Fig. 2D). As found in the sensory neurons, the synaptophysin-EGFP puncta were colocalized with the apPDE-FLAG signals in the sensory-to-motor synapses, indicating that apPDE was targeted to presynaptic sites (Fig. 2D).

\section{apPDE-specific $\mathrm{N}$-terminal domain was required for synaptic targeting}

We tested the role of the N-terminal domain of apPDE in targeting to the synaptic site. By using a mutant construct from which 
1-115 aa residues were deleted from the full length of apPDE (apPDE $\Delta \mathrm{N}$ ) (Fig. $3 A$ ), we compared its localization with that of the wild-type construct. Based on immunocytochemistry data, we analyzed colocalization between apPDE or apPDE $\Delta \mathrm{N}$ and synaptophysin by calculating the ratio between colocalized apPDE or apPDE $\Delta \mathrm{N}$ puncta and synaptophysin puncta (colocalization ratio; see Materials and Methods).

The N-terminal domain of PDE4 is known to be involved in membrane targeting (Shakur et al., 1993; Beard et al., 2002; Bolger et al., 2003). In accordance with this, apPDE $\Delta \mathrm{N}$ showed disrupted membrane localization and lower colocalization ratio than apPDE with synaptophysin puncta in distal neurites of sensory neurons (Fig. 3B). The average colocalization ratio of apPDE $\Delta \mathrm{N}$ was less than that of wild-type apPDE by $\sim 50 \%$ (apPDE, $0.99 \pm 0.01, n=7$ vs apPDE $\Delta \mathrm{N}, 0.53 \pm$ $0.08, n=14 ;{ }^{* *} p<0.01$, unpaired $t$ test), indicating that the apPDE-specific $\mathrm{N}$-terminal domain is critical for colocalization with synaptophysin puncta or synaptic targeting.

apPDE overexpression blocked synaptic facilitation in Aplysia sensory-to-

motor synapses

Because the elevation of cAMP level and activation of the PKA pathway are critical for 5-HT-induced synaptic facilitation in Aplysia (Castellucci et al., 1980, 1982; Bernier et al., 1982; Greenberg et al., 1987; Schacher et al., 1988; Byrne and Kandel, 1996), we investigated the role of apPDE in synaptic facilitation by overexpressing apPDE in Aplysia sensory-to-motor culture. Because apPDE had cAMP-specific phosphodiesterase activity (Fig. $1 A$ ) and was localized to synaptic regions (Fig. $2 D$ ), we speculated that manipulated expression of apPDE would alter the CAMP level in synaptic regions.

We microinjected the apPDE or control (EGFP) plasmid construct into the sensory neurons of the sensory-to-motor culture. Both short- and long-term synaptic facilitation induced by $5-\mathrm{HT}$ were significantly impaired when apPDE was overexpressed in the presynaptic sensory neurons (Fig. $4 A, B$ ). Short-term synaptic facilitation induced by 1 pulse of 5 -HT ( $5 \mathrm{~min}$ ) was considerably reduced by overexpression of apPDE $(17.9 \pm 4.9 \% ; n=6$; ${ }^{\star} p<0.05,{ }^{*} p<0.01$, unpaired $t$ test) (Fig. $\left.5 A, B\right)$ when compared with that in control cells expressing EGFP (51.6 $\pm 8.0 \%$; $n=5)$. Long-term facilitation induced by five pulses of 5 -HT was also significantly blocked by apPDE overexpression (18.1 \pm $4.1 \% ; n=6$ ) (Fig. $4 A, B$ ) when compared with that in control cells expressing EGFP (64.4 $\pm 7.0 \% ; n=5 ;{ }^{* *} p<0.01,{ }^{* * *} p<$ 0.001 , unpaired $t$ test) (Fig. 4A,B). However, apPDE overexpression did not affect basal synaptic transmission (percentage change, $8.4 \pm 1.7 \% ; n=6$ ), which was comparable with that of the control cells (EGFP-expressed cells, $10.5 \pm 6.6 \%$; $n=5$ ).
B
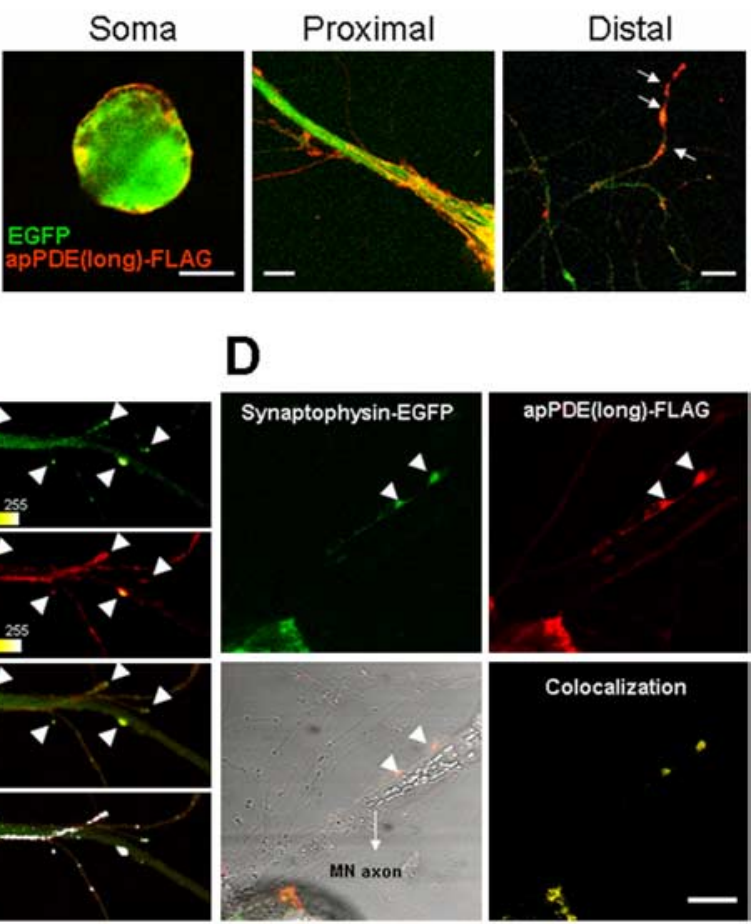

Figure 2. Membrane and synaptic localization of apPDE. $A$, To confirm the expression of apPDE in Aplysia sensory neurons, RT-PCR was performed with a specific primer set spanning the full length of apPDE and CDNA from pleural ganglia. - , RT mix only RT-PCR with the RT mixture that introduced reverse transcriptase $(+)$ produced a distinct band $(\sim 2.3 \mathrm{~kb})$, whic the expression of the apPDE mRNA in the Aplysia sensory neurons. $\boldsymbol{B}$, FLAG-tagged apPDE was microinjected with are the representative data from multiple experiments. In soma and proximal neurites, apPDE was mainly targeted to the memanalyze the localization of apPDE in the functional synapse, we cocultured pleural sensory neurons and abdominal LFS motor so construct sensory-to-motor synapses. The cultured neurons were immunostained $24-48 \mathrm{~h}$ after microinjection of the plasmid constructs. All of the cultured sensory-to-motor neurons demonstrate colocalization between synaptophysin-EGFP and apPDE-FLAG (arrowheads). Motor neuron (MN) axons are indicated (arrow). Scale bar, $20 \mu \mathrm{m}$.

These results suggest that apPDE overexpression in synaptic regions reduces 5-HT-induced long-term and short-term facilitation in Aplysia sensory- to-motor synapses probably by reducing the cAMP concentration.

Blockade of apPDE by RNA interference reduced synaptic plasticity in Aplysia sensory-to-motor synapses

To further examine the effect of cAMP regulation by apPDE on synaptic plasticity, we attempted to lower the expression level of apPDE by microinjecting apPDE-specific dsRNA into sensory neurons of the sensory-to-motor synapse. In our previous report, this method was found to inhibit gene expression specifically and effectively in Aplysia neurons (Lee et al., 2001, 2003). We performed immunocytochemistry to examine the inhibitory effect of apPDE dsRNA on the expression of apPDE. apPDE FLAG expression, as determined by anti-FLAG antibody staining, was significantly reduced in sensory neurons injected with apPDE dsRNA compared with that in cells injected with control luciferase dsRNA (dsLuci) (normalized fluorescence intensity, dsLuci injected, $0.78 \pm 0.16$; apPDE dsRNA injected, $0.34 \pm 0.06$ ) (sup- 
A

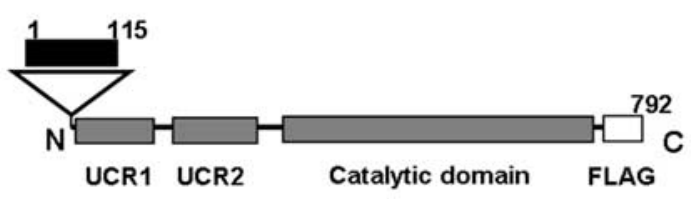

B
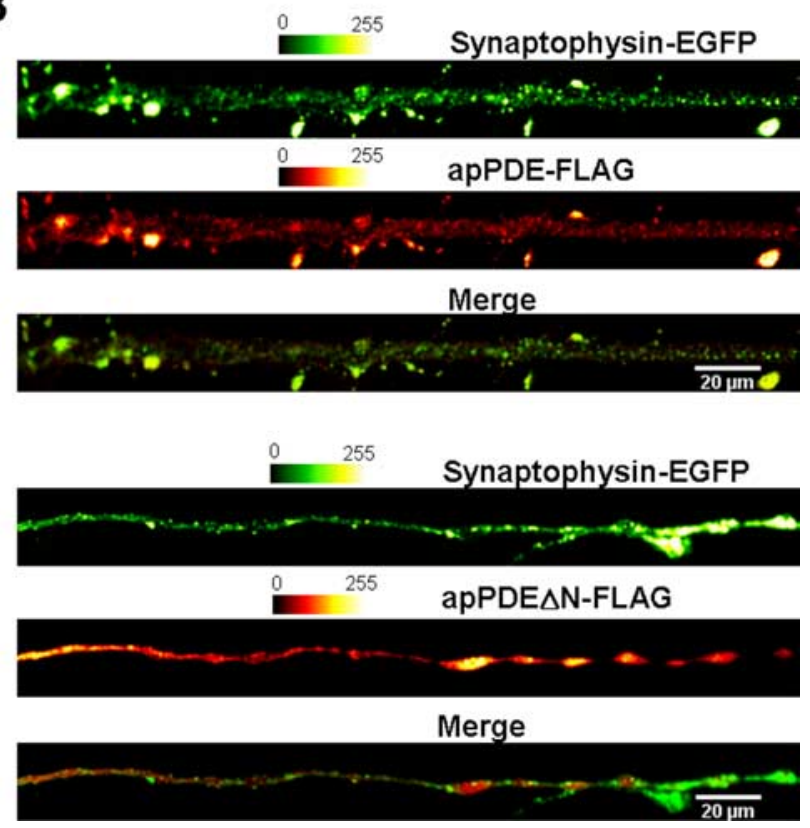

Figure 3. Unique $\mathrm{N}$-terminal domain of apPDE was essential for synaptic localization. $\boldsymbol{A}$, The structure of the apPDE-specific N-terminal domain-deleted mutant construct (apPDE $\Delta N$ ). Long-isoform-specific 1-115 aa residues were deleted from the full-length apPDE-FLAG constructs (see Materials and Methods). $\boldsymbol{B}$, Representative immunostained neurites of sensory neurons that were coexpressed with full-length apPDE-FLAG or apPDE $\Delta N-F L A G$ and synaptophysin-EGFP. Expression of apPDE $\Delta N-F L A G$ in sensory neurons showed disrupted membrane localization and colocalization with synaptophysin-EGFP. Each green and red image is shown with fluorescence intensity (0-255).

plemental Fig. 2, available at www.jneurosci.org as supplemental material).

It was observed that the introduction of apPDE dsRNA had no significant effect on the basal synaptic strength (percentage change, $-2.0 \pm 5.9 \% ; n=5$ ) compared with that of control synapses injected with luciferase dsRNA (percentage change, $4.2 \pm 2.1 \% ; n=8)$. On the contrary, cells injected with apPDE dsRNA produced neither short-term (percentage change, $7.7 \pm$ $6.6 \% ; n=6 ;{ }^{* *} p<0.001$, unpaired $t$ test) nor long-term (percentage change, $22.4 \pm 9.8 \% ; n=7 ;{ }^{\star} p<0.05$, unpaired $t$ test) facilitation (Fig. 4C,D). However, control cells injected with luciferase dsRNA showed normal synaptic facilitation (percentage change, short-term facilitation, $70.3 \pm 7.7 \%, n=4$; long-term facilitation, $70.4 \pm 15.1 \%, n=7$ ) (Fig. $4 C, D$ ). Together, these results indicate that reduced apPDE expression in synaptic regions blocks the 5-HT-induced short-term and long-term synaptic facilitation by disrupting the regulation of cAMP in Aplysia sensory-to-motor synapses.

\section{Overexpression of apPDE reduced presynaptic}

\section{PKA activation}

To examine the effects of apPDE overexpression or knock-down on the kinetics of the in vivo cAMP level attributable to 5-HT
A
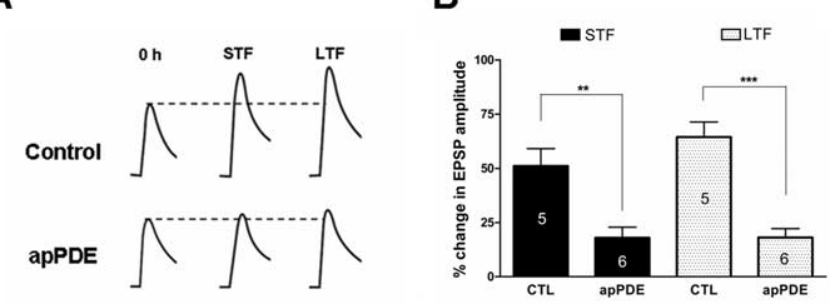

C

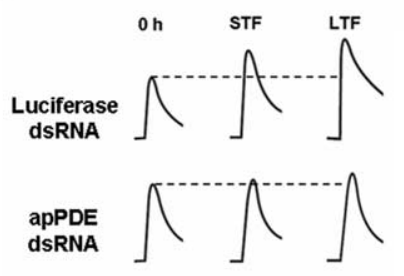

D

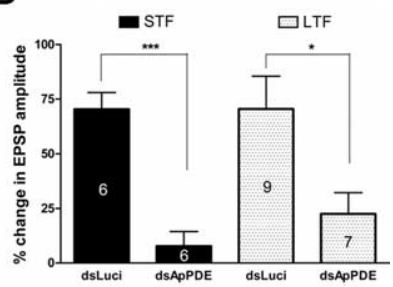

Figure 4. Impaired synaptic facilitation in Aplysia sensory-to-motor synapses by overexpression or RNAi of apPDE. To investigate the effect of apPDE overexpression or knock-down on short-term facilitation (STF) and long-term facilitation (LTF), the sensory-to-motor coculture was exposed to one pulse ( $5 \mathrm{~min}$ ) or five pulses of $10 \mu \mathrm{m} 5$-HT $24 \mathrm{~h}$ after microinjection of apPDE or dsRNA for apPDE. $A$, Representative EPSP recording traces in apPDE-overexpressed sensoryto-motor synapses. Overexpression of apPDE did not affect basal synaptic transmission (percentage change, $8.4 \pm 1.7 \% ; n=6)$, which was comparable with the synaptic strength of control cells $(10.5 \pm 6.6 \% ; n=5)$. However, both short-term facilitation produced by one pulse of 5-HT (10 $\mu \mathrm{m}$ ) as well as long-term facilitation produced by five pulses of 5-HT (10 $\mu \mathrm{m})$ were impaired in apPDE-overexpressed sensory neurons. $\boldsymbol{B}$, Bars represent the mean $\pm \mathrm{SEM}$ percentage change in the EPSP amplitude of the STF or LTF test. Control (CTL), STF, $51.6 \pm 8.0 \%$ $(n=5) ; \mathrm{LTF}, 64.4 \pm 7.0 \%(n=5)$ versus apPDE, STF, $17.9 \pm 4.9 \%(n=6) ; \mathrm{LTF}, 18.1 \pm 4.1 \%$ $(n=6) .{ }^{* *} p<0.01,{ }^{* *} p<0.001$, Student's $t$ test. $C$, Representative EPSP recording traces in apPDE knock-down sensory-to-motor synapses. The introduction of apPDE dsRNA had no significant effect on the basal synaptic strength (percentage change, $-2.0 \pm 5.9 \% ; n=5$ ), which was comparable with the synaptic strength of luciferase-dsRNA-injected cells as a control $(4.2 \pm 2.1 \% ; n=8)$. However, both STF and LTF were significantly reduced in apPDE dsRNAinjected sensory neurons. $D$, Bars represent mean \pm SEM percentage change in EPSP amplitude of STF or LTF. Control, STF, $70.3 \pm 7.7 \%(n=6)$; LTF, $70.4 \pm 15.1 \%(n=9)$ versus dsApPDE, STF, $7.7 \pm 6.6 \%(n=6) ;$ LTF, $22.4 \pm 9.8 \%(n=7) .{ }^{*} p<0.05,{ }^{* * *} p<0.001$, Student's t test.

stimulation, we performed in vivo measurement of PKA activation in living Aplysia sensory neurons using a modified FRET measurement between the regulatory and catalytic subunits of PKA (Bacskai et al., 1993; Zaccolo and Pozzan, 2002) (see Materials and Methods) (supplemental Fig. 3, available at www.jneurosci.org as supplemental material).

First, to determine the effect of apPDE overexpression on the PKA activation in synaptic region, apPDE-FLAG was coexpressed with RII-CFP and CAT-YFP in sensory neurons of the sensory-to-motor synapses. Because apPDE is highly localized to the presynaptic varicosities (Fig. $2 D$ ), the CFP/YFP ratio was measured in these regions. As shown in Figure 5A, the PKA activation and the stable maintenance of its elevated activity was observed in the control synapses after treatment with $10 \mu \mathrm{M}$ 5-HT (microinjected with empty vector). However, overexpression of apPDE resulted in a lowered PKA activity in the presynaptic varicosities than in control varicosities (Fig. $5 A$ ). This reduction in PKA activity attributable to apPDE overexpression was statistically significant (two-way ANOVA; $F=43.26$; $\mathrm{df}=1$; $p<0.01$ ). Individual comparisons with post hoc tests revealed that synaptic regions of EGFP (control)-expressed and apPDEoverexpressed cells showed that PKA activity at the all of the time points after 5 -HT treatment was significantly different ${ }^{*} p<$ 0.01 ) (Fig. 5A). This result indicates that apPDE could reduce the cAMP level in vivo. Moreover, this result suggests that impaired 
synaptic facilitation is attributable to the impaired PKA activation produced by the overexpression of apPDE in the synaptic regions.

Next, we investigated the effect of apPDE knock-down on cAMP kinetics. After microinjection of apPDE dsRNA with FRET sensor constructs (RII-CFP and CAT-YFP) into sensory neurons of the sensory-to-motor synapses, the FRET values were measured before and after treatment of $10 \mu \mathrm{M} 5$-HT at the presynaptic varicosities. In the control synapses, which were microinjected with luciferase dsRNA, treatment with $10 \mu \mathrm{M}$ 5-HT caused normal PKA activation (Fig. 5B). In the synapses injected with apPDE dsRNA, the initial PKA activation caused by 5 -HT was not considerably different from that in the control synapses (Fig. 5B); however, the PKA activity dropped to its basal level $90 \mathrm{~s}$ after 5-HT stimulation (Fig. 5B). A two-way ANOVA test indicated a significant effect of apPDE knockdown $(F=4.70$; $\mathrm{df}=1 ; p<0.05)$ on the PKA activity after 5-HT treatment. Individual comparisons with post hoc tests revealed that the synaptic region of luciferase dsRNA- and apPDE dsRNA-injected cells showed significantly different PKA activity $90 \mathrm{~s}$ after 5 -HT stimulation $\left({ }^{*} p<\right.$ 0.05 ) (Fig. $5 B$ ). We also performed PKA activation measurement using FRET to test whether exogenous cAMP treatment to apPDE knock-down synapses could rescue the impaired PKA activation. We found that the application of $200 \mu \mathrm{M} 8$-CPT-cAMP with 5-HT could activate PKA in apPDE knock-down synapses (supplemental Fig. 4, available at www. jneurosci.org as supplemental material), indicating that lowered PKA activity by injection of apPDE dsRNA (Fig. $5 B$ ) was attributable to neither the nonspecific effect of dsRNA on the FRET measurement nor a ceiling effect of the saturated cAMP level. Together, the knock-down of apPDE impairs the elevation of cAMP level induced by 5 -HT in synaptic regions, which might contribute to the impaired synaptic facilitation in the Aplysia sensory-to-motor synapses.

\section{Chronic rolipram treatment had an inhibitory effect similar to that of apPDE knock-down on cAMP kinetics}

Our results showed that inhibition of apPDE expression impaired the 5-HT-induced synaptic facilitation and PKA activation in the Aplysia sensory-to-motor synapses (Figs. 4D, 5B). However, treatment with rolipram, a PDE4-specific inhibitor, is known to enhance the hippocampal synaptic plasticity and memory by enhancing the cAMP levels (Barad et al., 1998). We hypothesized that these conflicting results might be attributable to the difference in the degree of PDE4 inhibition.

To test this hypothesis, we imitated the inhibitory effect of apPDE dsRNA by chronic treatment with rolipram. In Aplysia sensory neurons, $0.1 \mu \mathrm{M}$ rolipram was demonstrated to produce an increase in membrane excitability, which was regulated by cAMP-dependent inhibition of $\mathrm{K}^{+}$channels (Goldsmith and Abrams, 1992). To measure the effect of chronic inhibition of PDE4 on PKA activation, the sensory-to-motor cultures were
B

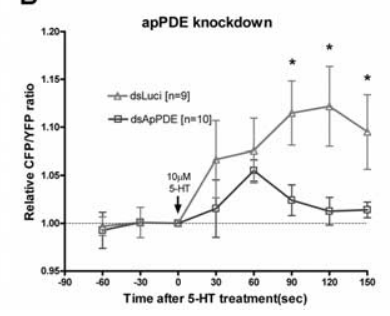

C

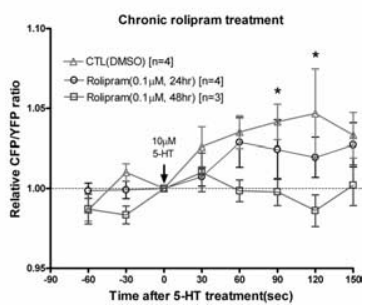

Figure 5. Dynamic change in PKA activation by 5-HT in apPDE-overexpressed, knock-down, and chronic PDE4-inhibited Surement results of apPDE-overexpressed synapses. Sensory neurons of sensory-motor cocultures were microinjected with ANOVA with post hoc tests. CTL, Empty vector-injected synapses; apPDE, apPDE-FLAG-injected synapses. $\boldsymbol{B}$, PKA activation mea列 theatment. Although the $24 \mathrm{~h}$-treated synapses illustrate a lower cAMP level than the DMSO-treated synapses, this difference is not significant ( $p>0.05$, two-way ANOVA with post hoc tests). However, a significantly lower cAMP level was observed in the $48 \mathrm{~h}$-treated synapses 60 and $90 \mathrm{~s}$ after 5 -HT treatment.

treated with $0.1 \mu \mathrm{M}$ rolipram for 24 and $48 \mathrm{~h}$ before 5 -HT treatment, and FRET values were measured after 5-HT stimulation. Overall, the PKA activation kinetics of 24 h-treated synapses decreased slightly, but there were no significant differences in the effect of 5-HT treatment between DMSO and rolipram (24 h treated) groups at all time points (two-way ANOVA, $p=0.39$ ) (Fig. $5 C$ ). In the case of the $48 \mathrm{~h}$-treated synapses, the PKA activity was significantly lower than that in the control synapses $60 \mathrm{~s}$ after the 5-HT treatment (two-way ANOVA; $F=8.70$; $\mathrm{df}=1.0$; $p<0.05$ ) (Fig. 5C). Individual comparisons with post hoc tests revealed that the DMSO and $48 \mathrm{~h}$ (rolipram) treated synaptic regions showed significantly different PKA activity from 90 to 120 s after the 5 -HT treatment $\left({ }^{\star} p<0.05\right)$ (Fig. 5C). These results suggest that chronic inhibition of PDE4 with rolipram impairs the increase in cAMP caused by the 5-HT in the synaptic regions; this is analogous to the effect of apPDE knock-down.

\section{Discussion}

apPDE is most probably close to a long isoform of mammalian PDE4D and targeted to presynaptic regions

Aplysia PDE showed high overall sequence homology with mammalian PDE4Ds (supplemental Fig. 1, available at www. jneurosci.org as supplemental material). This gene also showed a genetic structure, cAMP-specific substrate activity, and rolipram-sensitive inhibition of PDE activity similar to those of mammalian PDE4s (supplemental Fig. $1 A, B$, available at www.jneurosci.org as supplemental material) (Fig. $1 A, B)$.

Our immunocytochemistry data showed that apPDE is mainly targeted to the membrane region in soma and proximal neurites (Fig. $2 \mathrm{~B}$ ). This is consistent with other in vitro experi- 
ments with mammalian long-form PDE4, which reported that long-isoform PDE4s are detected in the membrane fraction (Bolger et al., 1997; Jin et al., 1998; Shepherd et al., 2003). apPDE was also highly distributed at synaptic regions in distal neurites colocalized with a synaptic marker, synaptophysin-EGFP (Fig. $2 C, D)$, similar to mammalian long-form PDE4A in the cerebellum (Shakur et al., 1995).

PKA subunits in Aplysia neuron also have the compartmentalized function and localization. For example, it has been known that type II regulatory subunit of PKA in Aplysia sensory neuron is also enriched in neurites in contrast to type I regulatory subunit in soma, and the expression level of each regulatory subunit is differentially affected by long-term facilitation (Liu et al., 2004). In a similar manner, short isoform of apPDE, which is found to be in Aplysia (see Materials and Methods), may contribute to the cAMP regulation in nonsynaptic region and have a distinct role for synaptic facilitation. Furthermore, we suggest that both apPDE and type II regulatory subunit of PKA may cooperate to regulate the PKA signaling cascade in the synaptic region because our results showed that synaptic PKA activity is tightly regulated by apPDE (Fig. 5A-C).

We also found that the long-isoform-specific N-terminal domain in apPDE is critical for synaptic targeting (Fig. $3 B$ ). However, we cannot exclude the possibility that other long-formspecific domains (UCR1 and UCR2) might be related with this localization because the colocalization ratio was not completely diminished by the deletion of this N-terminal domain (see Results). In concurrence with this, related mammalian PDE4 studies using chimeric constructs reported that UCR1 or UCR2 is also required for membrane targeting in non-neuronal cells (Shakur et al., 1993, 1995; Smith et al., 1996; Bolger et al., 1997; Jin et al., 1998; Beard et al., 2002; Shepherd et al., 2003). Future studies should be performed to find other molecular requirements for synaptic targeting of PDE4.

\section{apPDE regulates synaptic facilitation by regulating cAMP level in synaptic regions}

We analyzed the functional role of apPDE in synaptic facilitation through the overexpression or RNA interference (RNAi) of apPDE. Overexpressed apPDE did not affect the basal synaptic transmission; however, it did impair short-term and long-term synaptic facilitation (Fig. $4 \mathrm{~B}$ ). This might be the result of increased phosphodiesterase activity in the synaptic regions where apPDE was localized (Fig. 2D). Furthermore, knock-down of apPDE in the synaptic regions resulted in decreased synaptic facilitation without affecting the basal synaptic transmission (Fig. $4 D$ ). This result is consistent with the impaired learning and memory phenotype of the Drosophila dunce mutant (Dudai et al., 1976), $\mathrm{G}_{\mathrm{i}} \alpha$ knock-out mice (Pineda et al., 2004), and $\mathrm{G}_{\mathrm{s}} \alpha$ overexpressed Drosophila (Connolly et al., 1996). These data indicate that a balanced and optimized cAMP level regulated by $\mathrm{PDE} 4 \mathrm{~s}$ in synaptic regions is presumably important for synaptic plasticity and memory formation.

To test this possibility, we determined the relative change in the PKA activity caused by 5-HT in both apPDE-overexpressed and knock-down synapses. Because our in vivo imaging using FRET sensors represents the dissociation between regulatory and catalytic subunit (Bacskai et al., 1993; Zaccolo and Pozzan, 2002), indicating the degree of PKA activation, this technique allowed us to estimate the relative change in the in vivo PKA activity. We found that not only the overexpression but also the knock-down of apPDE impairs the PKA activation by 5-HT stimulation (Fig. $5 A, B)$. We also found that the treatment of a membrane- permeable cAMP analog activated the PKA in the apPDE knockdown synapses, indicating that reduced PKA activity in the apPDE knock-down synapses was not simply attributable to a ceiling effect of the saturated cAMP level in the apPDE knock-down synapses (supplemental Fig. 4, available at www.jneurosci.org as supplemental material). Therefore, we suggest two possible molecular mechanisms for this observation. First, chronic PDE inhibition may impair cAMP generation in response to external stimulation. A previous report has shown that the persistent $(>24 \mathrm{~h})$ activation of adenylyl cyclase in non-neuronal cells enhances the inhibitory pathway of adenylyl cyclase by the increase and decrease of the $G_{i} \alpha$ and $G_{s} \alpha$ expressions, respectively (Hadcock et al., 1990). Thus, PDE inhibition may cause the downregulation and/or desensitization of adenylyl cyclase in apPDE-inhibited sensory neurons. Second, it is possible that the inhibition of one isoform of PDE induces the expression of other PDE isoforms as a compensatory mechanism. Our data showed the rapid decay of PKA activation $(<90 \mathrm{~s})$ in response to 5-HT stimulation in apPDE-inhibited neurons, suggesting that there could be robust activities of other PDE isoforms. In the mammalian system, the activity of the short-isoform PDE4 is controlled by change in its expression level attributable to extracellular signals, such as elevated cAMP levels, although the activity of the long isoform of PDE4 is mainly modified by phosphorylation, ligand binding, and domain-domain interaction (D'Sa et al., 2002; Conti et al., 2003; Richter et al., 2005). Because the short isoforms of PDE4 were also found in Aplysia (data not shown; see Materials and Methods), these short isoforms are possibly involved in this compensatory mechanism in the PDE-inhibited cells. More studies will be required to clarify this question.

\section{Effects of chronic administration of rolipram on cAMP dynamics and synaptic facilitation}

The use of PDE4 inhibitors as promising drugs for ameliorating memory problems and several mental disorders, such as depression and anxiety, has been deliberated (Zhang et al., 2000, 2005; O'Donnell and Zhang, 2004). In contrast, other reports have also suggested that PKA overactivation by genetically manipulating animals or with the use of PKA activating agents would lead to impaired memory formation or instrumental learning (Baldwin et al., 2002; Pineda et al., 2004). Analogous to the results of PKA overactivation, inhibition of apPDE expression also impaired the synaptic facilitation in the Aplysia sensory-to-motor synapse (Fig. 4D). Our hypothesis was that these conflicting results arose from the different effects of the acute and chronic inhibitions of PDE4 on the intracellular cAMP levels. Because dsRNA effectively inhibits gene expression in Aplysia neurons over an extended period (Lee et al., 2001, 2003) (supplemental Fig. 2, available at www.jneurosci.org as supplemental material), the inhibitory effect of apPDE dsRNA is expected to be chronic when compared with that of acute PDE4 inhibitor treatment.

Chronic administration of rolipram, a PDE4 inhibitor, blocked the PKA activation caused by $5-\mathrm{HT}$, indicating impaired elevation of the cAMP level. This inhibition was more effective when the treatment with rolipram was prolonged (Fig. 5C). Therefore, chronic inhibition of the PDE4 activity or PDE4 downregulation in the synaptic sites could impair the cAMP homeostasis required for synaptic plasticity. These results suggest that the acute treatment with a PDE4 inhibitor would be more effective than chronic treatment to cure memory diseases or dementia.

In summary, we characterized the critical role of apPDE in cAMP kinetics during the synaptic facilitation process. apPDE 
downregulated the PKA activity in the synaptic region by reducing the cAMP level and showed the impaired synaptic facilitation when its normal expression level was disrupted. Moreover, our results suggest that the acute and chronic PDE4 inhibitions could lead to conflicting clinical effects in the treatment of memoryrelated diseases. To determine the precise molecular mechanisms governing cAMP kinetics and PDE4, future studies should be performed to characterize how each PDE4 isoform can differentially regulate the CAMP-dependent signaling and modulate the synaptic plasticity and memory.

\section{References}

Bacskai BJ, Hochner B, Mahaut-Smith M, Adams SR, Kaang BK, Kandel ER, Tsien RY (1993) Spatially resolved dynamics of cAMP and protein kinase A subunits in Aplysia sensory neurons. Science 260:222-226.

Baldwin AE, Sadeghian K, Holahan MR, Kelley AE (2002) Appetitive instrumental learning is impaired by inhibition of cAMP-dependent protein kinase within the nucleus accumbens. Neurobiol Learn Mem 77:44-62.

Barad M, Bourtchouladze R, Winder DG, Golan H, Kandel E (1998) Rolipram, a type IV-specific phosphodiesterase inhibitor, facilitates the establishment of long-lasting long-term potentiation and improves memory. Proc Natl Acad Sci USA 95:15020-15025.

Bauer AC, Schwabe U (1980) An improved assay of cyclic 3',5'-nucleotide phosphodiesterases with QAE-Sephadex columns. Naunyn Schmiedebergs Arch Pharmacol 311:193-198.

Beard MB, Huston E, Campbell L, Gall I, McPhee I, Yarwood S, Scotland G, Houslay MD (2002) In addition to the SH3 binding region, multiple regions within the $\mathrm{N}$-terminal noncatalytic portion of the cAMP-specific phosphodiesterase, PDE4A5, contribute to its intracellular targeting. Cell Signal 14:453-465.

Bernier L, Castellucci VF, Kandel ER, Schwartz JH (1982) Facilitatory transmitter causes a selective and prolonged increase in adenosine $3^{\prime}: 5^{\prime}$ monophosphate in sensory neurons mediating the gill and siphon withdrawal reflex in Aplysia. J Neurosci 2:1682-1691.

Bolger GB, Erdogan S, Jones RE, Loughney K, Scotland G, Hoffmann R, Wilkinson I, Farrell C, Houslay MD (1997) Characterization of five different proteins produced by alternatively spliced mRNAs from the human cAMP-specific phosphodiesterase PDE4D gene. Biochem J 328:539-548.

Bolger GB, McCahill A, Huston E, Cheung YF, McSorley T, Baillie GS, Houslay MD (2003) The unique amino-terminal region of the PDE4D5 cAMP phosphodiesterase isoform confers preferential interaction with beta-arrestins. J Biol Chem 278:49230-49238.

Byrne JH, Kandel ER (1996) Presynaptic facilitation revisited: state and time dependence. J Neurosci 16:425-435.

Castellucci VF, Kandel ER, Schwartz JH, Wilson FD, Nairn AC, Greengard P (1980) Intracellular injection of the catalytic subunit of cyclic AMPdependent protein kinase simulates facilitation of transmitter release underlying behavioral sensitization in Aplysia. Proc Natl Acad Sci USA 77:7492-7496.

Castellucci VF, Nairn A, Greengard P, Schwartz JH, Kandel ER (1982) Inhibitor of adenosine $3^{\prime}: 5^{\prime}$-monophosphate-dependent protein kinase blocks presynaptic facilitation in Aplysia. J Neurosci 2:1673-1681.

Chen CN, Denome S, Davis RL (1986) Molecular analysis of cDNA clones and the corresponding genomic coding sequences of the Drosophila dunce + gene, the structural gene for cAMP phosphodiesterase. Proc Natl Acad Sci USA 83:9313-9317.

Connolly JB, Roberts IJ, Armstrong JD, Kaiser K, Forte M, Tully T, O’Kane CJ (1996) Associative learning disrupted by impaired Gs signaling in Drosophila mushroom bodies. Science 274:2104-2107.

Conti M, Richter W, Mehats C, Livera G, Park JY, Jin C (2003) Cyclic AMPspecific PDE4 phosphodiesterases as critical components of cyclic AMP signaling. J Biol Chem 278:5493-5496.

D'Sa C, Tolbert LM, Conti M, Duman RS (2002) Regulation of cAMPspecific phosphodiesterases type 4B and 4D (PDE4) splice variants by cAMP signaling in primary cortical neurons. J Neurochem 81:745-757.

Dubnau J, Tully T (1998) Gene discovery in Drosophila: new insights for learning and memory. Annu Rev Neurosci 21:407-444.

Dudai Y, Jan YN, Byers D, Quinn WG, Benzer S (1976) dunce, a mutant of Drosophila deficient in learning. Proc Natl Acad Sci USA 73:1684-1688.

Fawcett L, Baxendale R, Stacey P, McGrouther C, Harrow I, Soderling S, Hetman J, Beavo JA, Phillips SC (2000) Molecular cloning and charac- terization of a distinct human phosphodiesterase gene family: PDE11A Proc Natl Acad Sci USA 97:3702-3707.

Francis SH, Turko IV, Corbin JD (2001) Cyclic nucleotide phosphodiesterases: relating structure and function. Prog Nucleic Acid Res Mol Biol 65:1-52.

Goldsmith BA, Abrams TW (1992) cAMP modulates multiple $\mathrm{K}^{+}$currents, increasing spike duration and excitability in Aplysia sensory neurons. Proc Natl Acad Sci USA 89:11481-11485.

Greenberg SM, Bernier L, Schwartz JH (1987) Distribution of cAMP and cAMP-dependent protein kinases in Aplysia sensory neurons. J Neurosci 7:291-301.

Hadcock JR, Ros M, Watkins DC, Malbon CC (1990) Cross-regulation between G-protein-mediated pathways. Stimulation of adenylyl cyclase increases expression of the inhibitory G-protein, Gi alpha 2. J Biol Chem 265:14784-14790.

Houslay MD, Adams DR (2003) PDE4 cAMP phosphodiesterases: modular enzymes that orchestrate signalling cross-talk, desensitization and compartmentalization. Biochem J 370:1-18.

Jin SL, Bushnik T, Lan L, Conti M (1998) Subcellular localization of rolipram-sensitive, cAMP-specific phosphodiesterases. Differential targeting and activation of the splicing variants derived from the PDE4D gene. J Biol Chem 273:19672-19678.

Karpova TS, Baumann CT, He L, Wu X, Grammer A, Lipsky P, Hager GL, McNally JG (2003) Fluorescence resonance energy transfer from cyan to yellow fluorescent protein detected by acceptor photobleaching using confocal microscopy and a single laser. J Microsc 209:56-70.

Kim JH, Udo H, Li HL, Youn TY, Chen M, Kandel ER, Bailey CH (2003) Presynaptic activation of silent synapses and growth of new synapses contribute to intermediate and long-term facilitation in Aplysia. Neuron 40:151-165.

Lee JA, Kim HK, Kim KH, Han JH, Lee YS, Lim CS, Chang DJ, Kubo T, Kaang BK (2001) Overexpression of and RNA interference with the CCAAT enhancer-binding protein on long-term facilitation of Aplysia sensory to motor synapses. Learn Mem 8:220-226.

Lee JA, Kim H, Lee YS, Kaang BK (2003) Overexpression and RNA interference of Ap-cyclic AMP-response element binding protein-2, a repressor of long-term facilitation, in Aplysia kurodai sensory-to-motor synapses. Neurosci Lett 337:9-12.

Liu J, Hu JY, Schacher S, Schwartz JH (2004) The two regulatory subunits of Aplysia cAMP-dependent protein kinase mediate distinct functions in producing synaptic plasticity. J Neurosci 24:2465-2474.

Martin KC, Michael D, Rose JC, Barad M, Casadio A, Zhu H, Kandel ER (1997) MAP kinase translocates into the nucleus of the presynaptic cell and is required for long-term facilitation in Aplysia. Neuron 18:899-912.

Mehats C, Andersen CB, Filopanti M, Jin SL, Conti M (2002) Cyclic nucleotide phosphodiesterases and their role in endocrine cell signaling. Trends Endocrinol Metab 13:29-35.

Montarolo PG, Goelet P, Castellucci VF, Morgan J, Kandel ER, Schacher S (1986) A critical period for macromolecular synthesis in long-term heterosynaptic facilitation in Aplysia. Science 234:1249-1254.

O'Donnell JM, Zhang HT (2004) Antidepressant effects of inhibitors of cAMP phosphodiesterase (PDE4). Trends Pharmacol Sci 25:158-163.

Pineda VV, Athos JI, Wang H, Celver J, Ippolito D, Boulay G, Birnbaumer L, DR. S (2004) Removal of G(ialphal) constraints on adenylyl cyclase in the hippocampus enhances LTP and impairs memory formation. Neuron 41:153-163.

Richter W, Jin SL, Conti M (2005) Splice variants of the cyclic nucleotide phosphodiesterase PDE4D are differentially expressed and regulated in rat tissue. Biochem J 388:803-811.

Schacher S, Proshansky E (1983) Neurite regeneration by Aplysia neurons in dissociated cell culture: modulation by Aplysia hemolymph and the presence of the initial axonal segment. J Neurosci 3:2403-2413.

Schacher S, Castellucci VF, Kandel ER (1988) cAMP evokes long-term facilitation in Aplysia sensory neurons that requires new protein synthesis. Science 240:1667-1669.

Sette C, Conti M (1996) Phosphorylation and activation of a cAMP-specific phosphodiesterase by the cAMP-dependent protein kinase. Involvement of serine 54 in the enzyme activation. J Biol Chem 271:16526-16534.

Shakur Y, Pryde JG, Houslay MD (1993) Engineered deletion of the unique $\mathrm{N}$-terminal domain of the cyclic AMP-specific phosphodiesterase RD1 prevents plasma membrane association and the attainment of enhanced 
thermostability without altering its sensitivity to inhibition by rolipram. Biochem J 292:677-686.

Shakur Y, Wilson M, Pooley L, Lobban M, Griffiths SL, Campbell AM, Beattie J, Daly C, Houslay MD (1995) Identification and characterization of the type-IVA cyclic AMP-specific phosphodiesterase RD1 as a membranebound protein expressed in cerebellum. Biochem J 306:801-809.

Shepherd M, McSorley T, Olsen AE, Johnston LA, Thomson NC, Baillie GS, Houslay MD, Bolger GB (2003) Molecular cloning and subcellular distribution of the novel PDE4B4 cAMP-specific phosphodiesterase isoform. Biochem J 370:429-438.

Smith KJ, Scotland G, Beattie J, Trayer IP, Houslay MD (1996) Determination of the structure of the N-terminal splice region of the cyclic AMPspecific phosphodiesterase RD1 (RNPDE4A1) by 1H NMR and identification of the membrane association domain using chimeric constructs. J Biol Chem 271:16703-16711.

Soderling SH, Beavo JA (2000) Regulation of cAMP and cGMP signaling: new phosphodiesterases and new functions. Curr Opin Cell Biol 12:174-179.

Soderling SH, Bayuga SJ, Beavo JA (1998a) Identification and characterization of a novel family of cyclic nucleotide phosphodiesterases. J Biol Chem 273:15553-15558.

Soderling SH, Bayuga SJ, Beavo JA (1998b) Cloning and characterization of a cAMP-specific cyclic nucleotide phosphodiesterase. Proc Natl Acad Sci USA 95:8991-8996.

Soderling SH, Bayuga SJ, Beavo JA (1999) Isolation and characterization of a dual-substrate phosphodiesterase gene family: PDE10A. Proc Natl Acad Sci USA 96:7071-7076.

Thompson WJ, Appleman MM (1971) Characterization of cyclic nucleotide phosphodiesterases of rat tissues. J Biol Chem 246:3145-3150.

Tully T, Quinn WG (1985) Classical conditioning and retention in normal and mutant Drosophila melanogaster. J Comp Physiol A Neuroethol Sens Neurol Behav Physiol 157:263-277.

Verghese MW, McConnell RT, Lenhard JM, Hamacher L, Jin SL (1995) Regulation of distinct cyclic AMP-specific phosphodiesterase (phosphodiesterase type 4) isozymes in human monocytic cells. Mol Pharmacol 47:1164-1171.

Wang H, Ferguson GD, Pineda VV, Cundiff PE, Storm DR (2004) Overexpression of type- 1 adenylyl cyclase in mouse forebrain enhances recognition memory and LTP. Nat Neurosci 7:635-642.

Zaccolo M, Pozzan T (2002) Discrete microdomains with high concentration of cAMP in stimulated rat neonatal cardiac myocytes. Science 295:1711-1715.

Zhang HT, Crissman AM, Dorairaj NR, Chandler LJ, O'Donnell JM (2000) Inhibition of cyclic AMP phosphodiesterase (PDE4) reverses memory deficits associated with NMDA receptor antagonism. Neuropsychopharmacology 23:198-204.

Zhang HT, Huang Y, Suvarna NU, Deng C, Crissman AM, Hopper AT, De Vivo M, Rose GM, O'Donnell JM (2005) Effects of the novel PDE4 inhibitors MEM1018 and MEM1091 on memory in the radial-arm maze and inhibitory avoidance tests in rats. Psychopharmacology (Berl) 179: 613-619. 